\title{
A Smart City Application: Waste Collection System with LoRaWAN Network for Providing Green Environment, Cost Effective and Low Power Consumption Solutions.
}

\section{Erol Aktay ( $\square$ erol.aktay@gazi.edu.tr)}

Gazi Üniversitesi https://orcid.org/0000-0001-6433-9722

Nursel Yalçın

Gazi University: Gazi Universitesi

\section{Research Article}

Keywords: Smart Waste Management, Internet of Things, LoraWan,Smart City, City Management, Lora LPWAN

Posted Date: April 26th, 2021

DOI: https://doi.org/10.21203/rs.3.rs-464675/v1

License: @ (i) This work is licensed under a Creative Commons Attribution 4.0 International License. Read Full License

Version of Record: A version of this preprint was published at IET Smart Cities on September 27th, 2021. See the published version at https://doi.org/10.1049/smc2.12014. 


\section{Abstract}

The trend towards cities and urbanization, which increases the number of people living in urban areas, makes it necessary for local authorities to be more efficient and effective in providing services and natural resources and to develop some strategies for a sustainable environment. The more effective use of resources, increasing awareness of sustainable environment, climate confidence and motivation to make cities more livable is a new concept Smart City. In this study, the proposed system supports the garbage collection of the city government and works with low budget, low energy and free radio frequencies. The loT (Internet of Things) sensor node is assembled, and the network is set up based on LoraWan protocol to connect it to the sample garbage can and collect data. Instant data collection by this network through the Internet of Things approaches and designed using this collected data. To establish an ideal system for smart garbage collection for cities and support sustainability in cities by integrating it with city government information systems. The data received from the sensor nodes and the efficiency of the system were demonstrated for the local governments. The primary outcome of this research is how to build a practical smart city application with minimum resources and support local authorities in their daily operation. Moreover, another objective is to investigate how low-power wan communication network in $868 \mathrm{Mhz}$ frequency works in istanbul- TURKEY and the best alternative for cellular networks for excellent communication in smart cities.

\section{Introduction}

The main objective in this study is to investigate the benefits of building a solid waste collection system based on IoT. IoT device will be based on LoRaWAN network which has many advantages in many aspects. With this study, extensive networks are created thanks to LoRaWAN technology which is not yet used in Turkey. In this study, the problem is studied from different perspectives. These are environmental issues, sustainability of cities, heavy dependence on fossil fuels for energy consumption in Turkey.

Before entering into the details of the study, the problems will be placed in the field in which the study would be of use. To this end, attention has been directed chiefly to the rate of growth of the population living in cities. The rapid population growth requires the use of loT to meet the demands of the population. On the other hand, the case of Turkey, its dependence on fossil fuels, which are currently preferred in disposal vehicles, as a country and its economic consequences are studied. Thirdly, in order to support the aim of the study, the increase of emission repairs over the years and its negative impact on the environment have been mentioned. At this point, emissions are mentioned to be reduced depending on the use of loT. Some of the issues such as effective management of natural resources, reducing environmental problems, supporting sustainable cities, cheaper solutions to urban problems, and reducing energy dependency serve as the source of motivation. When the details of routine garbage collection are examined, it becomes clear that there is a fixed collection route and period. This is no longer an effective way in today's world. Another solution is the low cost, emissions, need for fossil fuels and manpower required. Moreover, loT-assisted garbage collection provides a service that protects public safety, public health, and environmental quality.

In this model, It is expected to reduce labor-intensive activities frequency. Furthermore, the proposed system that creates an instant alert for garbage bin load. A dynamic system that response based on the data collected from the field is built. This will change scheduled collection service, decrease the need for more 
labor, and dramatically reduce service costs. In this model, the system is provided with LoRaWAN, which is an LPWAN protocol. LPWAN is more efficient in many ways from GSM-based solutions such as deployment cost and license. Based on Statistic's expectation in Fig. 1.1, the Number of LPWAN connections by technology worldwide from 2017 to 2023 will be dramatically increased. This is another indicator that LPWAN technologies will be more attractive.

In many loT projects, regardless of the type of connection, the power requirement is always the main subject. Power consumption of an loT product is a primer identifier; rather, it is suitable or not. The following Table 1.1 points out that LoraWan is thirty times more efficient in terms of power consumption for sleep current [2].

Table 1.1

Power Consumption

\begin{tabular}{|lll|}
\hline & LoRaWAN & NB-IoT \\
\hline TX Current & $24-44 \mathrm{~mA}$ & $74-220 \mathrm{~mA}$ \\
\hline RX Current & $12 \mathrm{~mA}$ & $46 \mathrm{~mA}$ \\
\hline Idle Current & $1.4 \mathrm{~mA}$ & $6 \mathrm{~mA}$ \\
\hline Sleep Current & $0.1 \mu \mathrm{A}$ & $3 \mu \mathrm{A}$ \\
\hline
\end{tabular}

\section{Internet Of Things}

Kevin Ashton, who is a technology pioneer, called loT term firstly in 1999. He described a system that the devices(objects) in this system connected through the Internet [3]. Connected sensors and devices (things) through the Internet mean the Internet of Things. IoT applications have been used increasingly for past decades, such as home automation (smart home), industrial process control, SCADA systems, waste management, measuring meters [4]. There are two fundamentals of loT. These are remote connectivity itself and the benefits of digitalized products and services for business. The loT is an essential subject, and demand for it increases dramatically. Opportunities such as controlling devices are offering plenty of solutions for both people and business [5]. Today, people live in coronavirus pandemic days and look for new devices to touch infected patients remotely and control the devices that support intensive care patients. This pandemic teaches people, companies, and governments to more interested in medical technologies also. loT applications will improve business and quality of life. IoT will change the way people live and work.

IoT offers many applications and convenient for various sectors. In health care, doctors can remotely control the patient's condition and take necessary action from afar. Furthermore, for the manufacturing sector, loT applications increase productivity. IoT makes precise tracking of the product manufacturing chain. It is also easy to measure the carbon emission for a plant. This may help to take action to reduce this emission in case of any regulation violation. [6]. In most applications, loT infrastructure is divided into some stages. These stages are listed briefly, Application Layer, Network Layer, and Edge Layer. These three-layered infrastructures are an accepted model usually in Edge Layer, where data is collected via sensors and devices. The network 
layer is the communication part that is necessary to post data to the endpoint (edge layer). The application layer is the last part and, in this layer, provide service and many applications to the users [7].

\section{Smart Cities And Sustainability}

The expectation in UN World Urbanization Prospects is renewed regularly, and estimating \%70 of the world's total population will live in urban areas. The rising population and effective resource management smart city have become mandatory objects for cities [8]. An evolving solution to deal with this scenario is the convergence of information and communication technologies by implementing the smart cities' concept. The smart cities concept is the pioneering solution for supplying higher living standards. To provide such higher living standards, coverage of Information and Communication technologies must be extended through every point of a city [9].

From the historical perspective, there are some different indicators when the smart city concept was first used. In the late 1990s, the main parts of the smart city concept, city, and ICT, started to discuss academic literature. In 1997, more than 2000 cities had web pages (www), and today this is accepted as a sort of the first example of the smart city concept [10]. The smart city concept emerged from the development of ICT [11]. Some other best practices represent milestones for the smart city concept. Some of them are Amsterdam, Copenhagen, Seoul, and New York City [12].

Global Data Thematic Research published research and listed the significant milestones in the smart city concept [13]. According to the research first evidence of the smart city, the concept was the big data project for Los Angeles in 1974.

The development of the smart city concept requires some other developments in areas such as ICT, IoT, and sensor networks. When All these parts are associated, the ideal system would be in service for people. This whole extensive system can be named as a cluster of some hardware and software. This cluster works intensively to understand the needs of people and respond in time. Not only people or resident of a city, but this cluster also support industries, city management, and government. [14].

ISO (International Standardization Organization) published a new standard in 2018 (ISO 37120:2018 Sustainable cities and communities-Indicators for city services and quality of life). This guidance standard will be leading the future smart city projects and support the decision-making process. Briefly standard suggest that; Cities need indicators to measure their performance. Indicators and test methods mentioned in this standard are for supporting city management to;

- Measure the performance of the city service and quality of life.

- Compare measurements and create values based on these measurements.

- Provide more data that help policymakers in the decision-making process.

Indicators included in ISO37120 are listed in this standard [15].

\section{Wireless Transmission For lot}


Internet of things systems requires the transmission layer. Some alternative communication technologies are in stock for loT systems. Some of them LoRaWan, SigFox, NB-loT, 5G, wi-fi, ZigBee. One of them is LoraWan, and it offers many features for low-cost and long-range communications. Most loT projects deploy LoraWan into their network [4]. In this paper, a solution which is LoraWan based will be proposed.

The LoRa Alliance is an open, nonprofit association that has grown to more than 500 members since its inception in March 2015, becoming the most significant and fastest-growing alliance in the technology sector. Its members closely collaborate and share experiences to promote and drive the success of the LoRaWAN protocol as the leading open global standard for secure, carrier-grade loT LPWAN connectivity. In the definition of the LoRa Alliance, "The LoRaWAN specification is a Low Power, Wide Area (LPWA) networking protocol designed to wirelessly connect battery operated 'things' to the internet in regional, national or global networks, and targets essential Internet of Things (IOT) requirements such as bi-directional communication, end-to-end security, mobility and localization services." [16].

Long Range accessibility and low-power requirement make LPWA a more desired network communication method for loT infrastructures. LPWA offers some advantages when it is compared to other alternative network technologies. LPWA provides efficient signal propagation in indoor places that the cellular system does not correctly work. LPWA uses MHZ frequency band, for Europe $867-869 \mathrm{MHz}$, North America 902-928 MHz, China 470-510 MHz, Korea 920-925 MHz, Japan 920-925 MHz, India 865-867 MHz LPWA meets the requirement for long-distance communication applications. If the sensor nodes are attached to the garbage bin, a city LPWA fits needs. In such waste management applications, the data rate is not high, and delaytolerant is acceptable, so LPWA is an ideal transmission method for this loT system. The most crucial advantage of LPWA is saving more energy and connecting the endpoint node sensor directly to the gateway via star topology [17].

Low Power; LPWA provides both mesh topology and star topology. If it is planned a long-range communication for large areas, mesh topology helps extend the coverage of LPWA. By this method, more 10 $\mathrm{km}$ coverage could be reached. For an endpoint device, it is expected to be live for a long time as ten years. Without dying battery, keep network live is thanks to a star topology. Every endpoint node connects straight to the gateway, and this brings more efficiency in terms of power need. Long-Range Communication: LPWA's better signal propagation enables the signals to reach indoor places, so end to end device connection rage changing from a few $\mathrm{km}$ to 10 kilometers depending on the environment (building and plants block signals and decrease the distance) Low Cost; In most scenario, LPWA uses unlicensed frequency band. In Turkey, LPWA works in $868 \mathrm{MHz}$ in ISM Band.

The Industrial, Scientific, and Medical (ISM) frequency bands are designated radio frequency bands defined by the ITU Radio Regulations. ISM band refers to some spectrums reserved for scientific, medical, and industrial requirements rather than communications. There is not a spectrum cost, and this makes LPWA more attractive [17].

\section{Essential Problems Should Be Addressed For Turkey: Sustainability, Greenhouse Gas Emission And Energy Dependency Rate.}


Year by year, people intend to live in cities for better living conditions [18]. United Nations expectation is that the $\% 68$ population will live in urban areas by 2050 , and It means that the population in urban areas will increase from $\% 55$ to $\% 68$. United Nations 2050 expectation also shows that 2.5 billion people will be added to urban areas population, and a significant part of this increase will be taking place in Asia and Africa [19].

It also should be considered that people can easily find a job in cities compared to job opportunities in rural areas. Furthermore, many jobs require sitting on the desk all day. On the contrary, people should do much more muscular activities while working in rural areas. Not only more job availability make cites more attractive. People can reach high-speed Internet, shopping centers, more public transportation facilities, and food in cities [18]. These are the significant factors supporting the increasing trend of living in cities and the growing population.

Turkey has been experiencing environmental pressures due to population growth, industrialisation and rapid urbanisation. The following Table 2.1 below shows that the number of people living in rural and urban areas based on the years. It is not hard to consider that both population and the number of people living in urban areas increased dramatically.

Table 2.1

Number of people living in urban and rural areas in Turkey [20]

\begin{tabular}{|llllll|}
\hline Year & $\begin{array}{l}\text { Total } \\
\text { Population }\end{array}$ & $\begin{array}{l}\text { Urban } \\
\text { Population }\end{array}$ & $\begin{array}{l}\text { Rural } \\
\text { Population }\end{array}$ & $\begin{array}{l}\text { Urban } \\
\text { Population }\end{array}$ & Rural Population \\
\hline 1927 & 13.648 .270 & 3.305 .879 & 10.342 .391 & 24.2 & 75.8 \\
\hline 2017 & 80.810 .525 & 74.761 .132 & 6.049 .923 & 92.5 & 7.5 \\
\hline
\end{tabular}

The number shown on the table that millions of people live in huge cities because of this, many chaotic problems occur. Air pollutions, management of cities, and usage of natural resources are some of them. Table 2.2 shows that how much fossil energy resources that TURKEY needed in the past year. The numbers on the table demonstrate some realities. When people moved to cities from rural areas, and TURKEY's development increased year by year, energy needs were boosted too during the past years. These facts result in economic and environmental troubles.

Table 2.2

Turkish Statistical Institution

Petrol Import Report

\begin{tabular}{|lll|}
\hline Years & Metric & Total \\
\hline 2009 & Ton & 14.219 .427 \\
\hline 2019 & Ton & 25.820 .442 \\
\hline
\end{tabular}

Another energy resource is electricity that mainly produced by using fossil fuels, coal, and natural gas. In this case, there is more waste heat, so producing energy from fossil fuels is not an efficient way. Besides this efficiency problem, fossil fuels also release much more pollution into the air; this creates health issues for people [21]. 
In our times, it is widely recognized that the economic and social development depends on the protection of the environment and reduction of the human impact. Urbanization has been bringing some problems to people's life and emerging some new issues. Green gas emission inventory has been present for Turkey since 1990. The Green gas emission inventory is pretty new when it is compared to some other countries. Fossil Energy demand has been rising owing to industrialization and urbanization. In 2016 green gas emission is \%135.4 is higher than in 1990. In 1990 it was about 210 million tons of greenhouse gas emissions, but today it is almost 495 million tons. It is expected that: The rate of 1990 will dramatically increase to $467 \%$ in 2030 , and total emission cis also reached up to 1175 million tons [33]. Following Fig. 2.1 shows the greenhouse gas emission rate from 1990 to 2018.

$\mathrm{CO} 2$ emission is not the only leading cause of air pollution. $\mathrm{N} 2 \mathrm{O}$ emissions, $\mathrm{CH} 4$ emissions, rice production, natural gas, and oil production are other sources of the problem. Additional environmental pollution is plastic waste. Based on some measurements and data, In the EU region, one person produces $10-40 \mathrm{~kg}$ of plastic waste yearly, and the average is $31 \mathrm{~kg}$ per person. In some research, plastic waste has become a severe problem for the human food chain. Whether air pollution or plastic waste, sustainability must be considered increasingly, and more technology should be used to support a sustainable environment [22]. New technologies and the speed of network connections in ICTs have been created extraordinary abilities for both people and businesses. It has become the most important for sustainable cities and urbanization [24].

\section{Research Design And Methodology}

In the previous sections, some information is provided and tried to find an answer for the following questions,

- Why do people move to urban areas from rural areas?

- What makes urban areas more attractive?

- What are urbanization and the consequences of urbanization?

- What are the main issues for creating sustainable cities?

- What is the Internet of things, and how does it work?

- What are the smart city and sustainable smart cities?

- What are the alternatives for data transmission in smart cities?

- What are the LPWA and advantages of LPWA?

moreover, some topics, urbanization, environmental issues such as pollutions, the Internet of things, smart city and sustainably, and LPWAN for loT transmission are examined.

Against the background of the information from the previous sections, this section presents the research data and findings. One of the significant problems faced by local authorities is effective solid waste management is discussed. At this point, following topics will be enlightened in this research;

- The traditional waste collection method is no longer suitable. It cannot cope with current problems because of operating costs and environmental problems, such as air pollution, waste disposal on the street and quality of service. 
- Operation cost is expected to shrinkage dramatically based on new approach which decreases the need of labor force, operation equipment's (tracks)

- No need for data subscription or licensing cost to send the data.

- The population of Istanbul, Turkey's largest city, is larger than that of 131 countries in the world, according to the United Nations Population Fund [25]. A survey applied to Istanbul will be an insightful delivery for upcoming application and research.

- At the time of the on-site research, there was no significant $868 \mathrm{Mhz}$ LoraWan application in Istanbul or Turkey. The research results will show us that Lora devices can operate on $868 \mathrm{Mhz}$ in Turkey.

- Some computational metrics are examined to prove that reducing the waste collection load results in less demand for labor, fossil energy, and operating equipment, and avoids more carbon emissions.

The Internet of things infrastructure will be integrated into the municipality's current waste management service and anticipate finding solutions for the problems listed above that conventional waste collection operations cannot find proper and modern solutions. For the fieldwork, official research permission is received from the Beşiktaş Municipality "Beşiktaş has extraordinary historical heritage, and it is mostly called Istanbul city center and located on the European side of Istanbul. Many commercial companies and residential located in Besiktas. Besiktas is also serving as a hub for transportation to many directions in İstanbul." [26] to conduct the research study. Furthermore, Arnavutkoy Municipality also provided valuable support for some open area tests. In this research, the endpoint node is built by assembling the following hardware. General information about each hardware gets from the producer company and shared briefly below

\subsection{Components of IOT end point and infrastructure}

- Semtech SX1276 (137 MHz to 1020 MHz Long Range Low Power Transceiver): The SX1276/77/78/79 transceivers feature the $\mathrm{LoRa}{ }^{\circledR}$ long range modem that provides ultra-long range spread spectrum communication and high interference immunity while minimizing current consumption [27].

- LG01-P IoT Gateway: The LG01-P is an open-source single channel LoRa Gateway. It lets bridge LoRa wireless network to an IP network via WiFi, Ethernet, Or 3G/4G cellular via optional LTE module. The LoRa wireless allows users to send data and reach extremely long ranges at low data-rates. It provides ultralong range spread spectrum communication and high interference immunity [28].

- Arduino Uno: Arduino Uno is a microcontroller board based on the ATmega328P and takes place in various loT projects [29].

- HC - SR04 Ultrasonic Ranging Module: Ultrasonic ranging module HC - SR04 takes place in some loT project and It provides distance measurement service. It can measure the distance from $2 \mathrm{~cm}$ to $400 \mathrm{~cm}$ in good accuracy. There are both ultrasonic receiver, transmitter, and control circuit included in this module [30].

- ESP8266 Module Adapter: This module is mandatory as an adapter for Semtech sx1276. Sx1276 should be soldered to this module to work correctly with other peripherals.

- Other Subsidiary Equipment for Node Design: Breadboard, Antenna, USB Cable, 9V Battery, Battery Connector, Solid Core Jumper Wires, Jumper Wires, Plastic Project Box, Solder, Electric Soldering Iron, 
Screwdriver (For Mounting), Screw (For Mounting), Hydraulic Gas Support Shock Absorber Arm, Hot Silicone Gun and Stick (For Box Implementation)

The block diagram shown in the following Fig. 6.1 represents the physical links that connect components within the system.

\subsection{Software and Programing}

Arduino Uno Arduino Software, Mac OSX version 1.8.9, used for programming. The Arduino sketch uploaded to the Arduino is accessible from "https://github.com/eaktay/LoraWan.git". Details and essential points that should be considered to build a proper node in this code will be given in the next sections. To make this code working on the hardware above, many tests are done. When the literature is investigated, there was no similar design, so all circuits were perpetually tested for days and made in Turkey. The sample code is from the GitHub library [31], and it is configured based on needs. It is shown in the following pictures that all parts are assembled final stage of the node and the primary process of assembling. In Picture 6.1, the entire development environment is shown. The local development was made successfully, thanks to these products. Picture 6.2 is demonstrating how to attach the distance sensor to the node box. Ultrasonic sensor, Lora module, and Arduino board connection made, as shown in picture 6.3. Connecting the Lora module, the Arduino board is not comfortable without a supportive board shown in picture 6.4 Lora module should be soldered to this assistance board in advance.

After all necessary assembling processes are completed, all parts of the node implemented into the box, as shown in picture 6.5 .

After the assembly of the node was completed, it was connected to the trash can for testing. In this scenario, an ordinary trash can on the street was used. It was meeting the standard that is European Committee for Standardization EN 840-2. In picture 6.6, this attachment is shown. The main purpose of this implementation is to keep the loT node healthy during the opening and closing times of the damper. Another reason for this setup was to absorb the shock of closing the flap of the container. The flap weighs too much, which can speed up the closing motion and destroy the node, as shown in Fig. 6.7, which attaches the node to the top of the flap.

The reason for that to keep the node clean and make the accuracy more. Picture 6.8, 6.9, and 6.10 show the whole stand by bin with the node's implementation. QR code is added for providing more information on the web.

\subsection{Management of the Operation and Hardening the System}

After the node is deployed in the field, the system is started, and data is collected from the nodes. In the previous it was mentioned that the code is uploaded to the Arduino Uno board. In this code, many indicators and parameters are configured. First of all, the distance is $30 \mathrm{~cm}$ for the ultrasonic sensor distance data read from the node. This means that when the city residents put the solid waste in the bin and the height of this waste approaches the top, less than $30 \mathrm{~cm}$, the node sends a message (data) and informs the central system that the bin will soon be full. How this works technically, ultrasonic sensors send ultrasound into the bin, and 
when the sound wave is reflected, the ultrasonic sensor measures the distance and sends this data to the card Arduino Uno. At this point, the process can be referred to as the edge layer.

The next step is the transmission phase, where the Arduino board sends the data to the central database. The Dragino gateway is introduced in the previous sections. The gateway receives the data from the node and transmits it to the next phase. In this project, The Things Network infrastructure is used to collect, store and process data from the field. The Things Network is an open-source initiative that operates an open Internet of Things infrastructure supported by a global ecosystem of thousands of developers, IT integrators, hardware manufacturers, universities, and governments. To achieve data transfer from the endpoint to the central database, all devices must be configured. It is used for the node side, and some parameters that Arduino uses to forward data are inserted.

First, the gateway is registered on the TTN network. The gateway is configured through its GUl and the parameters are set to forward data to the TTN network. Once the gateway receives data from the node, it forwards all the data to the TTN network servers. While configuring the Arduino, the keys are inserted in the Arduino code such as access key, gateway ID, gateway key APPLICATION EUIS, Activation Method, Device EUI, Application EUI, Device Address, Network Session Key, App Session Key, which TTN provides. For all these settings, the following website was visited. Some of the screenshots of TNN web pages are shown below.

https://account.thethingsnetwork.org/users/logi n

Picture 6.12 TTN registration page

After logging into the TTN website, Picture 6.12 appears. On this page, a gateway must first be set up. In this scenario, the dragino gateway is registered in the TTN. Via the maintenance interface of the gateway, it is not difficult to set up a gateway for communication with TTN. Once the gateway is registered in the TTN, the page shown in Picture 6.13 displays details of the gateway registration.

Picture 6.13 Registered Gateway Profile

When the gateway is registered, the infrastructure is also provided. Thanks to the communication between the gateway and TTN, it will be convenient to send data from the endpoint node to the central database of TTN. Picture 6.14 is the data monitoring screen. When the Arduino board collects data, it sends it to the TTN, and the data flow would be monitored on the following screen.

Picture 6.14 QR Code for More Information

TTN allows the developer to integrate their system with TTN systems. In this application, the integration is set up to post data to the webserver to monitor it on the website. Picture 6.15 is the page of the HTTP integration configuration.

Picture 6.15 TTN HTTP Integration Configuration for Data Posting

All the information that is needed should be defined on this page. In this configuration on the node, gateway, and TTN, data is collected from the field. Data does not mean everything independently. An alarm should be 
set and an ideal workflow for waste collection should be designed. When the data shows up on the TTN, it is forwarded to the web server via HTTP integration. TTN provides such functions. On the web server the location of the bin is stored in advance.

For regular garbage collection, the best route is created and the need to find empty bins is avoided. To facilitate this process, an app is being developed for Firebase's IOS platform. Firebase is an application development platform from Google that provides powerful tools for creating a mobile app. Thanks to this application, the operation of the work would be easily done by using IOS map algorithm and either setting the location of the bin or creating the best route will not be a headache. The sample video on using the IOS app uploaded to the cloud is available at the following link https://www.dropbox.com/s/tjv821fra2qs53l/da5ba8fd-a392-4c47-b55b-e5178dc74bfb.mp4?dl=0

The following screenshots are the samples from application progress.

\section{Picture 6.16 Login Screen Picture 6.17 New Node Adding}

\section{Picture 6.18 Node List Picture 6.19 Route Planning}

When the IOS application is opened, the screen in picture 6.16 appears. After describing the credentials, the application searches for the location to add a new note, as shown in picture 6.17. Since the infrastructure of GPS is used, the location data is completely correct. In the following steps, meaningful information is added to determine the node, as shown in picture 6.18. The node identity information is inserted into the Arduino Uno code and uploaded to the node. When the node pushes data to the TTN, the load is realized in the node, then the collection process is queued based on the best route. The value of distance is set to $30 \mathrm{~cm}$ by default. When the height of the garbage load reaches this value, the node sends data to the TTN because the node is full. More frequent reading and sending to the TTN network consume more energy, so the frequency is four hours a day. Different districts in Besiktas and Arnavutköy were selected to provide data for the system. For this purpose, a site visit is planned with the officials of Besiktas and Arnavutköy Municipalities. Thanks to this visit, it is possible to estimate how this system would efficiently manage some cost and environmental issues.

The location of the bin and its load are recorded, and this data is then posted to a map, shown in picture 6.18. When the loT node is implemented at all garbage bins, these green bins will no longer need to be hit, saving money and reducing air pollution. All user service vehicles that serve the purpose of garbage collection use fossil oil, and as mentioned in the previous section, this causes air pollution.

This study shows that the LoraWan technology and protocol are applicable in Turkey. It is achieved to mount a pattern node and point-to-point on LPWAN network. Collecting data from the field is achievable and this supports the idea that if the same nodes are implemented in a wide area, this will reduce the expenditure on the traditional garbage collection method. When all the information from the field visits, observations and the proposed loT system are taken into account, the proposed model will definitely reduce the cost of waste management by at least $40-45 \%$. This rate is estimated by operating costs such as fuel, employees and trucks. 
Another fact is that if this new garbage collection system is used by most communities in Turkey, this will also maintain a clean environment. In addition, it is estimated that the initial savings of $40-45 \%$ in operation will result in less need for fossil energy. This will be an opportunity to reduce dependencies and positively affect the foreign trade deficit.

\section{Picture 6.20 Sample Waste Collection Operation Map}

Figure In picture 6.20 shows the study area. This area is called Gayrettepe. The field was visited with municipality officials to understand how the system for garbage collection works. During this visit, it was noted that some of the garbage bins were not full and should not be included in the collection queue. The number of full and empty bins provides an indication of the extent to which the proposed system creates economic and sustainable value for the environment.

One of the main purposes of the field visits is to understand the operational process of garbage collection and to find the weak points of the current garbage collection system. This field visit provided valuable information such as where the sensor node should be placed, which bin locations should not be visited, and how to develop a well-designed garbage collection system. In some cases, the garbage collection system should be triggered due to environmental issues. That is, if some garbage bins are full before the regular time, these bins should be emptied to maintain a healthy and pleasant environment for city residents.

Authentication, trust, and integrity of the communication channel between loT devices are the critical security parameters that modern solutions deal with. Although the loT is up to date, it lacks the support of powerful devices and is not compatible enough to deal with the increasing heterogeneous entities [32]. To harden the entire IOT infrastructure, some security parameters are mandatory. To make the entire system more secure, there are several issues that need to be considered during implementation and also during operation. Some of them are: PKI/digital certificates, network security, API security, network access control, segmentation, security gateways, patch management, continuous software updates, training and consumer education.

\section{Conclusions, Implications, And Future Directions}

This article proposes a LoraWan-supported smart city waste management. In this article, the problems are clearly expressed. These are environmental problems, population, lack of ICT based systems, cost of operation. In this article, before offering the solution, the current situation in Turkey is considered in terms of greenhouse gas emission, cost of fossil fuel import which affects the country's budget, and cost of waste collection for local authorities. Several important issues are examined; the proposed solution will address all of them. Based on the field visits and observations, traditional trash collection is no longer satisfactory. Given the questions: why does the garbage truck go to all the garbage cans that are not full? Why do garbage trucks have to travel more miles for daily garbage collection? Why is more fossil fuel needed to operate? Why are more people hired to handle the daily trash pickup? In order to create a sustainable environment, reduce the fossil fuel import budget and reduce the expenditure on garbage collection, a new approach is developed.

In this study, LoraWan wireless technology is the first application in Turkey. The design of nodes, network configuration, application-level management and operational process are properly regulated. Instead of the current licensed spectrum and services, an unlicensed free ISM band is proposed. The innovative parts of this 
proposal are low cost and low power applications. Once the node is connected to the bins, there will be no GSM data charges and higher battery requirements. This is a pioneering approach.

For future studies, all bins should be empowered by other sensor nodes. This means that there is an existing infrastructure; thanks to this infrastructure, other sensors such as humidity, air quality, sound, weight could be connected to the node. In this way, the government agencies in charge of city management will get a significant power to build a fully smart city.

The establishment of sensor cluster will provide important data and information to the local authorities to make the cities more sustainable. The main problem faced during the research is the limited resources, so only some nodes are built to show its efficiency and working process. In future studies, there could be support from companies or policy makers to extend the scope of the study.

\section{Abbreviations}

Notations used for this paper.

\begin{tabular}{|c|c|c|c|}
\hline MHz & Megahertz & $\mathrm{dB}$ & Decibel \\
\hline TTN & The Things Network & $\mathbf{V}$ & Volt \\
\hline LoraWan & Long Range Wide Area Network & kbps & Kilo Bit Per Second \\
\hline ISM & The Industrial, Scientific, and Medical & $\mathbf{P A}$ & Power Amplifier \\
\hline LPWA & Low Power Wide Area & $\mathrm{mA}$ & Mili Amper \\
\hline IoT & Internet of Things & $\mathbf{R X}$ & Receive \\
\hline $\mathrm{CO} 2$ & Carbon Dioxide & nA & Nano Amper \\
\hline NO2 & Nitrogen dioxide & $\mathrm{Hz}$ & Hertz \\
\hline CH4 & Methane & FSK & Frequency-Shift Keying \\
\hline EU & European Union & GFSK & Gaussian Frequency Shift Keying \\
\hline ICT & $\begin{array}{l}\text { Information and Communications } \\
\text { Technology }\end{array}$ & MSK & Minimum-Shift Keying \\
\hline ITU & $\begin{array}{l}\text { International Telecommunication } \\
\text { Union }\end{array}$ & GMSK & Gaussian Minimum-Shift Keying \\
\hline GSM & $\begin{array}{l}\text { The Global System for Mobile } \\
\text { Communications }\end{array}$ & OOK & On-Off Keying \\
\hline M2M & Machine to Machine & LTE & Long-Term Evolution \\
\hline RFID & Radio-Frequency Identification & 3G & $\begin{array}{l}\text { The Third Generation of Wireless } \\
\text { Communications }\end{array}$ \\
\hline UN & United Nations & 4G & $\begin{array}{l}\text { The Fourth Generation of Wireless } \\
\text { Communications }\end{array}$ \\
\hline ISO & $\begin{array}{l}\text { The International Organization for } \\
\text { Standardization, }\end{array}$ & LAN & Local Area Network \\
\hline NB-IoT & Narrow Band IoT & GUI & Graphical User Interface \\
\hline 5G & $\begin{array}{l}\text { The Fifth Generation of Wireless } \\
\text { Communications }\end{array}$ & SSH & Secure Shell \\
\hline $\mathbf{R F}$ & Radiofrequency & DC & Direct Current \\
\hline $\mathrm{dBm}$ & decibel-milliwatts & TTL & Time to Live \\
\hline www & World Wide Web & & \\
\hline
\end{tabular}

\section{Declarations}


All authors certify that they have no affiliations with or involvement in any organization or entity with any financial interest or non-financial interest in the subject matter or materials discussed in this manuscript.

\section{References}

[1]. Number of LPWAN connections by technology worldwide from 2017 to 2023 . Retrieved from https://www.statista.com/statistics/880822/lpwan-ic-market-share-by-technology/. Last access (25.04.2020).

[2]. LoRaWAN vs NB-loT: A Comparison Between loT Trend-Setters. Retrieved from https://ubidots.com/blog/lorawan-vs-nb-iot/. Last access (27.05.2020).

[3]. R. H. Weber, E. Studer. Cybersecurity in the Internet of Things: Legal aspects. Computer law \& security review, 32, (2016), 715-728.

[4]. M. Eldefrawy, I. Butun, N. Pereira, M. Gidlund. Formal security analysis of LoRaWAN. Computer Networks, 148, (2019), 328-339.

[5]. T. Saarikko, U. H. Westergren, T. Blomquist. The Internet of Things: Are you ready for what's coming? Business Horizons, 60, (2017), 667-676.

[6]. 12 A. Louchez. The future of the Internet of Things will be 'EPIC'. Retrieved from https://news.itu.int/internet-of-things-iot-epic-future/. Last Access (20.04.2020).

[7]. $\quad 13$ H. HaddadPajouh, A. Dehghantanha, R. M. Parizi, M. Aledhari, H. Karimipour. A survey on internet of things security: Requirements, challenges, and solutions. Internet of Things, November 13, (2019), 21-19.

[8]. C. Colldahl, S. Frey, J. E. Kelemen. (2013). Smart Cities: Strategic Sustainable Development for an Urban World. (Master's Thesis). Blekinge Institute of Technology, Karlskrona, Sweden.

[9]. P. Marques, D. Manfroi, E. Deitos, J. Cegoni, R. Castilhos, J. Rochol, E. Pignaton, R. Kunst. An loT-based smart cities infrastructure architecture applied to a waste management scenario. Ad Hoc Networks, 87, (2019), 200-208.

[10]. L.G. Anthopoulos, Understanding Smart Cities: A Tool for Smart Government or an Industrial Trick Public Administration and Information Technology 22,DOI 10.1007/978-3-319-57015-0_2

[11]. T. Brandt, W. Ketter, B. Donnellan and R T. Watson. AIS Pre-ICIS Workshop on "IoT \& Smart City Challenges and Applications", ISCA, 2016.

[12]. L. G. Anthopoulos, P. Fitsilis. Evolution Roadmaps for Smart Cities: Determining Viable Paths. Conference: 13th European Conference on eGovernment, ECEG, June 2013.

[13]. History of smart cities: Timeline. Retrieved from https://www.verdict.co.uk/smart-cities-timeline/. Last access $(23.05 .2020)$ 
[14]. G. Fortino, L. Fotia, F. Messina, D. Rosaci, G.M.L. Sarné. A meritocratic trust-based group formation in an loT environment for smart cities. Future Generation Computer Systems, 108, (2020), 34-45.

[15]. ISO 37120:2018 (en) Sustainable cities and communities, Indicators for city services and quality of life. Retrieved from https://www.iso.org/obp/ui/\#iso:std:iso:37120:ed-2:v1:en. Last Access (20.04.2020).

[16]. About LoRa Alliance. Retrieved from https://lora-alliance.org/about-lora-alliance. Last Access (20.04.2020).

[17]. F. Muteba, K. Djouani, T. Olwal. A comparative Survey Study on LPWA IoT Technologies: Design, considerations, challenges and solutions considerations, challenges and solutions. Procedia Computer Science, 155, (2019), 636-641.

[18]. Ozdemir, E. (2018). Modernism, Urbanization, and Turkey. Akdeniz University, Social Science Institution Journal, 2018(4), 79-96.

[19]. Türk, S. ve Çalışkan, H. Effect of the Development in Urbanization on Economy: Turkey Example. (2019). Iğdır University Social Science Journal, 17, 677-694.

[20]. Department of Economic and Social Affairs. The world population projected to live in urban areas by 2050, Retrieved from https://www.un.org/development/desa/en/news/population/2018-revision-of-worldurbanization-prospects.html. Last Access (15.04.2020).

[21]. Turkish Statistical Institute. Turkey's fossil fuel imports per year. Retrieved from http://www.tuik.gov.tr/PreTablo.do?alt_id=1046, Last Access (20.04.2020).

[22]. S. Nizetic, N. Djilali, A. Papadopoulos, J. J. P. C. Rodrigues. Smart technologies for promotion of energy efficiency, utilization of sustainable resources and waste management. Journal of Cleaner Production. Journal of Cleaner Production, 231, (2019), 565-591.

[23]. Turkish Statistical Institute. Greenhouse Gas Emission. Retrieved from http://www.tuik.gov.tr/PreTablo.do?alt_id=1019. Last Access (20.04.2020).

[24]. S. E. Bibri. A foundational framework for smart sustainable city development: T Theoretical, disciplinary, and discursive dimensions and their synergies. Sustainable Cities and Society, 38, (2018), 758794.

[25]. Istanbul's population more than 131 countries: UN body, Retrieved from https://www.aa.com.tr/en/economy/istanbul-s-population-more-than-131-countries-un-body/1421496. Last access (15.04.2021).

[26]. History of Besiktas. Retrieved from http://en.besiktas.bel.tr/category/history-of-besiktas/. Last Access (20.04.2020).

[27]. Semtech Wireless RF Product. Retrieved from https://www.semtech.com/products/wireless-rf/loratransceivers/sx1276. Last access (20.04.2020). 
[28]. The Dragino LG01-P loT Gateway Features. Retrieved from https://www.dragino.com/products/loralorawan-gateway/item/117-Ig01-p.html. Last access (20.04.2020).

[29]. Arduino Uno datasheet. Retrieved from https://store.arduino.cc/usa/arduino-uno-rev3. Last access (20.04.2020).

[30]. HCSR04 sensor datasheet. Retrieved from https://cdn.sparkfun.com/datasheets/Sensors/Proximity/HCSR04.pdf. Last access (20.04.2020)

[31]. Arduino Sample Code. Retrieved from https://github.com/dragino/ArduinoProfileExamples/blob/master/libraries/Dragino/examples/LoRa/LoRaWAN/Arduino_LMIC/Arduino_LMIC.ino. Last access (20.04.2020).

[32]. S. Bhatt, Rachit, P.R Ragiri. Security trends in Internet of Things: a survey, (2020.

[33]. M. M. Yatarkalkmaz, M. B. Özdemir. The calculation of greenhouse gas emissions of a

\section{Figures}

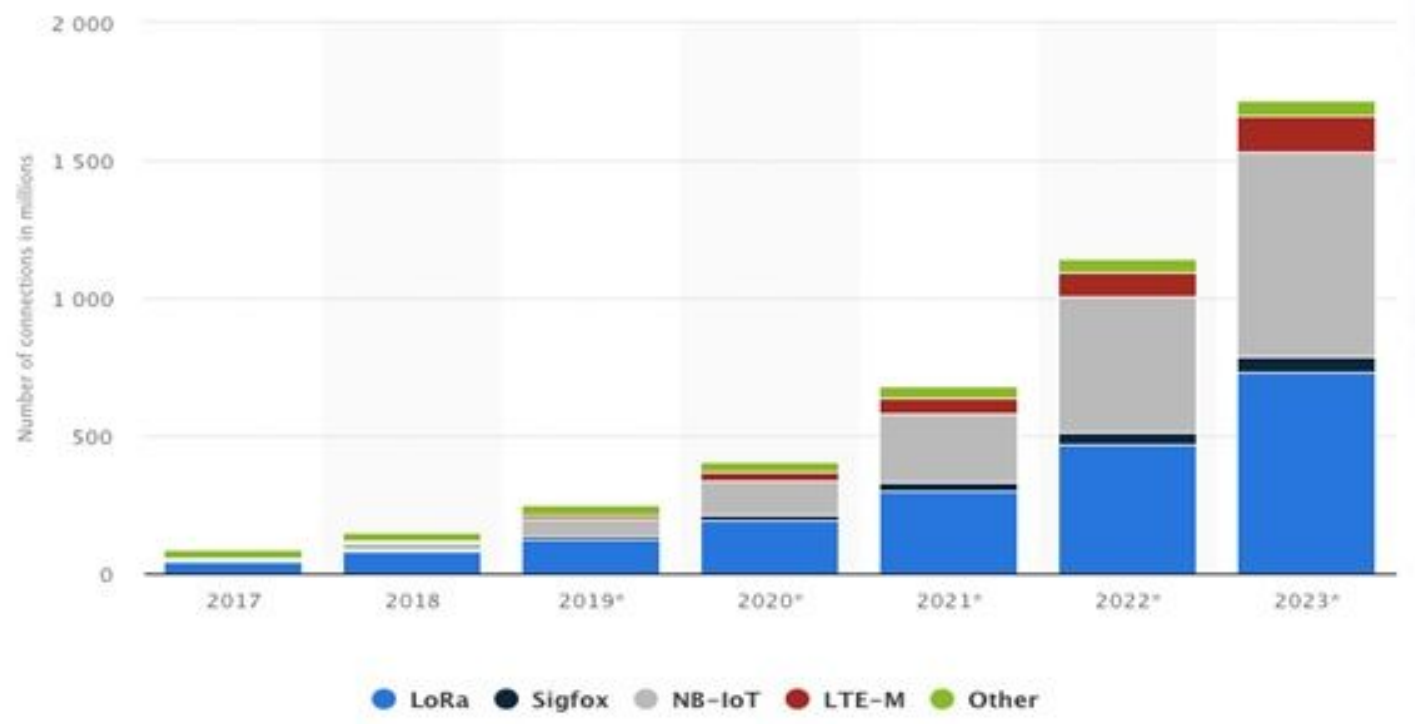

\section{Figure 1}

Figure 1.1 LPWAN Connections by Technology Worldwide From 2017 to 2023, [1]. 


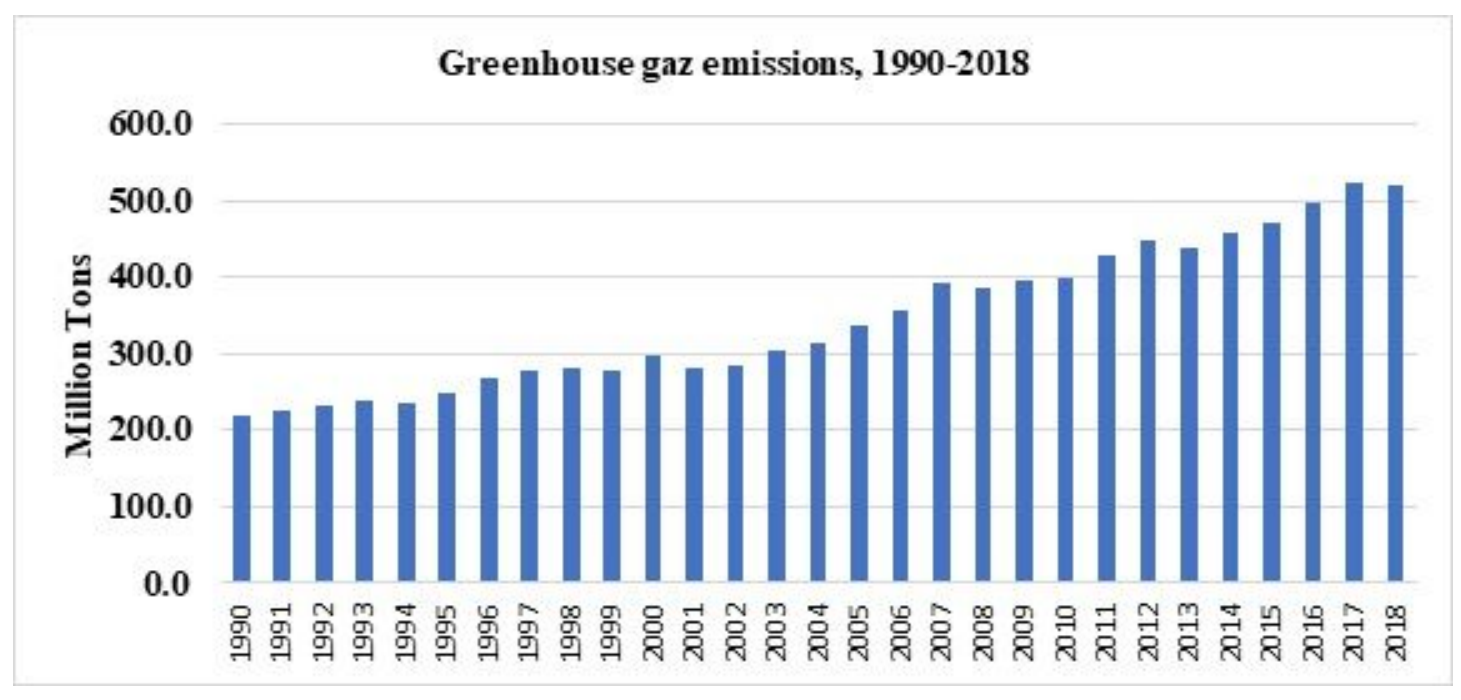

Figure 2

Figure 2.1 Greenhouse gas emission in Turkey [23]

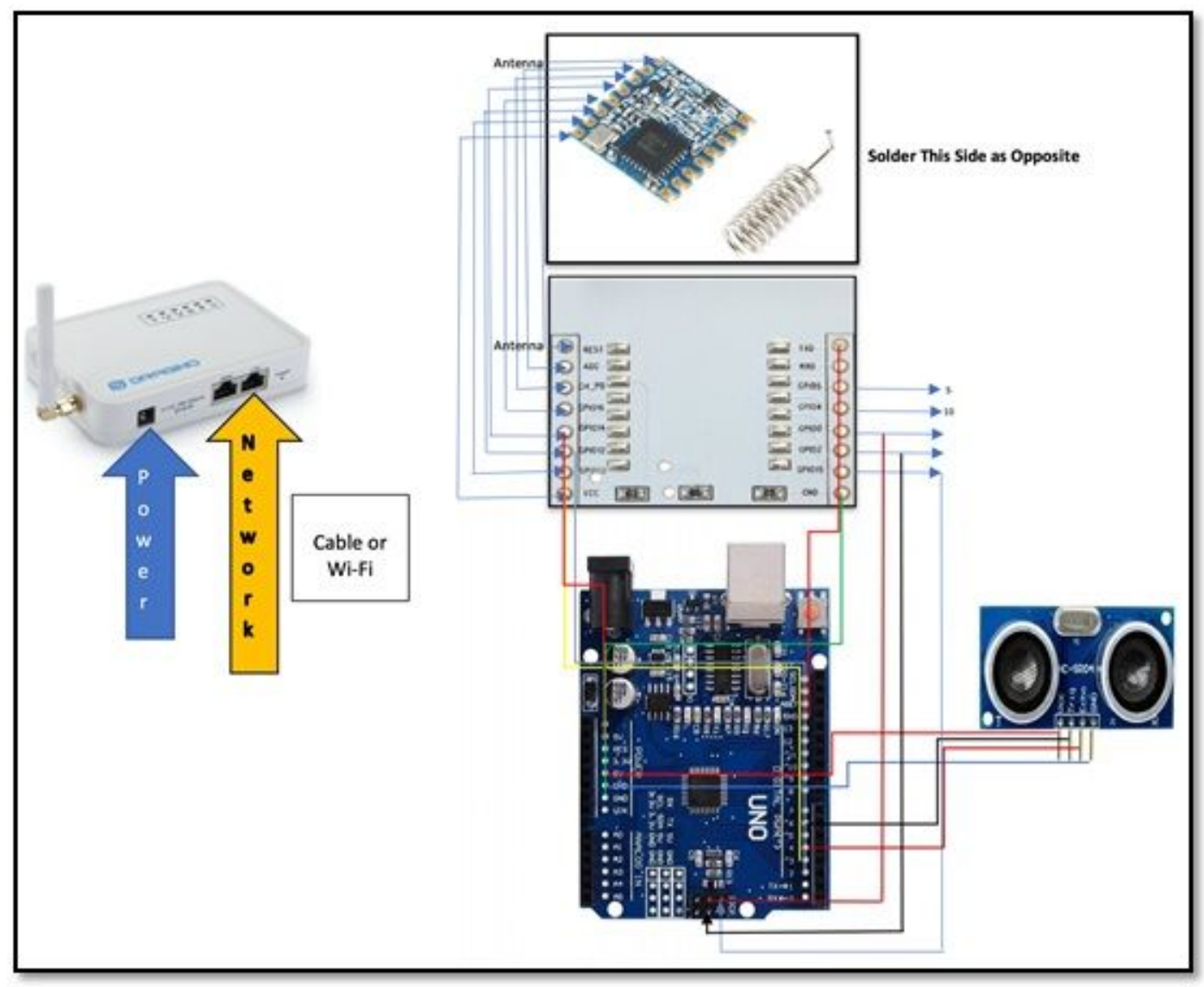

Figure 3

Figure 6.1 Block Diagram 


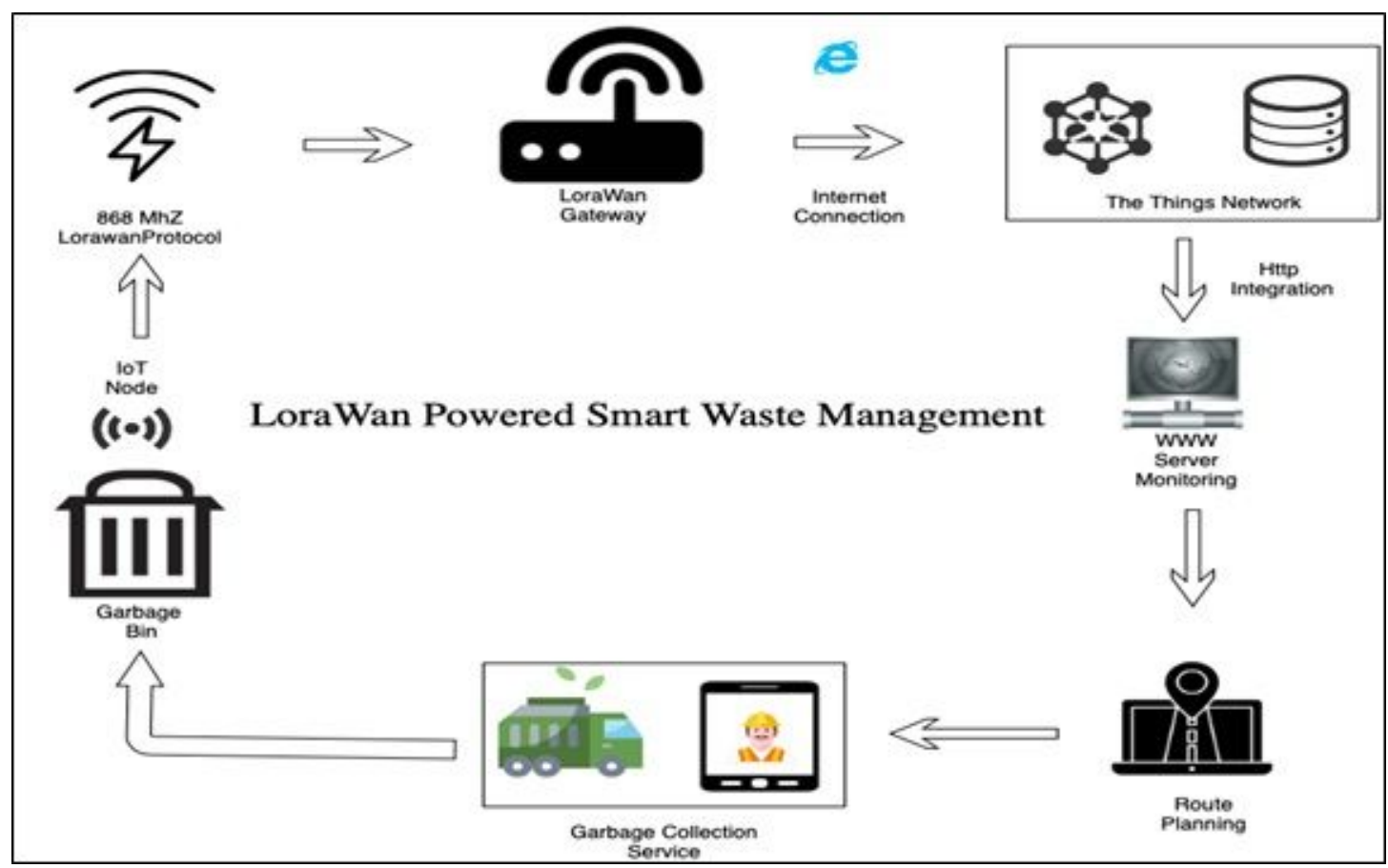

Figure 4

Figure 6.2 Overall Processes in the Infrastructure

\section{Supplementary Files}

This is a list of supplementary files associated with this preprint. Click to download.

- Pictures.pdf 